\title{
Risk assessment in pulmonary arterial hypertension and chronic thromboembolic pulmonary hypertension
}

\author{
Marc Humbert (10 ${ }^{1,2,3}$, Harrison W. Farber ${ }^{4}$, Hossein-Ardeschir Ghofrani ${ }^{5,6}$, \\ Raymond L. Benza ${ }^{7}$, Dennis Busse ${ }^{8}$, Christian Meier ${ }^{9}$ and Marius M. Hoeper (1) ${ }^{10}$ \\ Affiliations: ${ }^{1}$ University Paris-Sud, Faculté de Médecine, Université Paris Saclay, Le Kremlin-Bicêtre, France. \\ ${ }^{2}$ AP-HP, Service de Pneumologie, Centre de Référence de l'Hypertension Pulmonaire, Hôpital Bicêtre, Le \\ Kremlin-Bicêtre, France. ${ }^{3}$ INSERM Unité 999, Le Kremlin-Bicêtre, France. ${ }^{4}$ Division of Pulmonary, Critical \\ Care and Sleep Medicine, Tufts Medical Center, Boston, MA, USA. ${ }^{5}$ University of Giessen and Marburg Lung \\ Center, Member of the German Center for Lung Research (DZL), Giessen, Germany. ${ }^{6}$ Dept of Medicine, \\ Imperial College London, London, UK. ${ }^{7}$ Cardiovascular Institute, Allegheny General Hospital, Pittsburgh, PA, \\ USA. ${ }^{8}$ Chrestos Concept GmbH \& Co. KG, Essen, Germany. ${ }^{9}$ Bayer AG, Berlin, Germany. ${ }^{10}$ Clinic for \\ Respiratory Medicine, Hannover Medical School, Member of the German Center for Lung Research (DZL), \\ Hannover, Germany.
}

Correspondence: Marc Humbert, Service de Pneumologie, Hôpital Bicêtre, 78 rue du Général Leclerc, 94270 Le Kremlin-Bicêtre, France. E-mail: marc.humbertđaphp.fr

@ERSpublications

Riociguat improves risk group in patients with PAH and CTEPH according to three abbreviated versions of the ESC/ERS risk stratification model (results of a post hoc analysis) http://ow.ly/ Herk30o81KM

Cite this article as: Humbert M, Farber HW, Ghofrani H-A, et al. Risk assessment in pulmonary arterial hypertension and chronic thromboembolic pulmonary hypertension. Eur Respir J 2019; 53: 1802004 [https://doi.org/10.1183/13993003.02004-2018]

\section{ABSTRACT}

Background: Current pulmonary hypertension treatment guidelines recommend use of a risk stratification model encompassing a range of parameters, allowing patients to be categorised as low, intermediate or high risk. Three abbreviated versions of this risk stratification model were previously evaluated in patients with pulmonary arterial hypertension (PAH) in the French, Swedish and COMPERA registries. Our objective was to investigate the three abbreviated risk stratification methods for patients with mostly prevalent $\mathrm{PAH}$ and chronic thromboembolic pulmonary hypertension (CTEPH), in patients from the PATENT-1/2 and CHEST-1/2 studies of riociguat.

Methods: Risk was assessed at baseline and at follow-up in PATENT-1 and CHEST-1. Survival and clinical worsening-free survival were assessed in patients in each risk group/strata.

Results: With all three methods, riociguat improved risk group/strata in patients with PAH after 12 weeks. The French non-invasive and Swedish/COMPERA methods discriminated prognosis for survival and clinical worsening-free survival at both baseline and follow-up. Furthermore, patients achieving one or more low-risk criteria or a low-risk stratum at follow-up had a significantly reduced risk of death and clinical worsening compared with patients achieving no low-risk criteria or an intermediate-risk stratum. Similar results were obtained in patients with inoperable or persistent/recurrent CTEPH.

Conclusions: This analysis confirms and extends the results of the registry analyses, supporting the value of goal-oriented treatment in PAH. Further assessment of these methods in patients with CTEPH is warranted.

This article has supplementary material available from erj.ersjournals.com

Received: Oct 192018 | Accepted after revision: March 122019

Copyright $\odot$ ERS 2019. This article is open access and distributed under the terms of the Creative Commons Attribution Non-Commercial Licence 4.0. 


\section{Introduction}

Pulmonary arterial hypertension (PAH) and chronic thromboembolic pulmonary hypertension $(\mathrm{CTEPH})$ are subtypes of pre-capillary pulmonary hypertension, a rare but debilitating condition $[1,2]$. Despite improvements in the short-term survival of patients with $\mathrm{PAH}$, the condition remains incurable. By contrast, CTEPH is potentially curable by pulmonary endarterectomy (PEA) [1-5]; however, up to $50 \%$ of patients are considered inoperable and up to $51 \%$ develop persistent/recurrent pulmonary hypertension after undergoing PEA [6-11].

For patients with PAH, the 2015 European Society of Cardiology (ESC)/European Respiratory Society (ERS) treatment guidelines and the Proceedings of the 2018 World Symposium on Pulmonary Hypertension recommend regular comprehensive risk assessment at expert pulmonary hypertension centres using a range of clinical, echocardiographic, exercise, biochemical and haemodynamic parameters $[1,12]$. As such, patients can be categorised as low, intermediate or high risk, with an estimated 1-year mortality of $<5 \%, 5-10 \%$ and $>10 \%$, respectively. Achieving and maintaining a low-risk profile is the recommended goal of PAH treatment $[1,12]$.

Recently, three abbreviated versions of the 2015 ESC/ERS risk stratification model have been evaluated retrospectively in newly diagnosed (incident) PAH cohorts of the French, Swedish and COMPERA registries [13-15]. The French registry invasive method classifies patients according to the number of ESC/ ERS low-risk criteria present for World Health Organization (WHO)/New York Heart Association (NYHA) functional class (FC), 6-min walk distance (6MWD), right atrial pressure (RAP) and cardiac index. The French registry non-invasive method classifies patients according to the number of ESC/ERS low-risk criteria present for WHO/NYHA FC, brain natriuretic peptide (BNP)/N-terminal pro-BNP (NT-proBNP) and 6MWD. The Swedish/COMPERA method grades 6MWD, WHO FC, NT-proBNP, RAP, cardiac index and mixed venous oxygen saturation $\left(\mathrm{SvO}_{2}\right)$ from 1 to 3 (1=low, 2=intermediate, $3=$ high) using the ESC/ERS risk thresholds. The rounded mean of these grades is then used to define patient risk stratum. Each model demonstrated a favourable prognostic outcome for patients with a greater number of low-risk criteria or patients in the "low-risk" strata versus other strata, at both baseline and follow-up [13-15].

There is currently no recommendation regarding how to assess risk in patients with inoperable CTEPH; however, in addition to its common and well-validated use in assessing risk in patients with PAH, the REVEAL risk score (RRS) has shown utility as a prognostic tool in patients with CTEPH [16] and a recent study suggested that the Swedish/COMPERA method predicts mortality in non-operated patients with CTEPH [17].

Riociguat is a soluble guanylate cyclase stimulator [18-22] approved for the treatment of both PAH and inoperable or persistent/recurrent CTEPH [23-28]. In the 12-week PATENT-1 study, riociguat significantly improved exercise capacity and a range of secondary end-points in patients with mostly prevalent PAH [24]. Similar benefits of riociguat were observed in patients with inoperable or persistent/ recurrent CTEPH in the 16 -week CHEST-1 study $[23,24]$. The improvements in $6 \mathrm{MWD}, \mathrm{WHO}$ FC and NT-proBNP observed in the PATENT-1 and CHEST-1 studies were maintained at 2 years of riociguat treatment in the long-term extension studies PATENT-2 and CHEST-2 [25-28]. Patients in both studies were well characterised by invasive and non-invasive assessment at baseline and at the end of the double-blind study period, with patients being followed for a median of 139 weeks in PATENT-2 and 116 weeks in CHEST-2.

These studies provided an opportunity to conduct a post hoc analysis of the three abbreviated risk assessment methods in patients with mostly prevalent PAH and inoperable or persistent/recurrent CTEPH to explore the association between patient risk group or stratum, before and after riociguat treatment, and long-term outcomes.

\section{Methods}

\section{Patients and studies}

PATENT-1 (ClinicalTrials.gov identifier NCT00810693) was a 12-week, phase III, double-blind study in which patients with PAH were randomly assigned to placebo, riociguat individually dose adjusted to a maximum of $2.5 \mathrm{mg}$ three times daily or an exploratory dose of $1.5 \mathrm{mg}$ riociguat three times daily (not included in the risk assessment analyses). CHEST-1 (ClinicalTrials.gov identifier NCT00855465) was a 16-week, phase III, double-blind study in which patients with inoperable CTEPH or persistent/recurrent pulmonary hypertension were randomly assigned to placebo or riociguat individually adjusted to a maximum of $2.5 \mathrm{mg}$ three times daily. Patients completing PATENT-1 and CHEST-1 without ongoing study drug-related serious adverse events were eligible to enter the PATENT-2 (ClinicalTrials.gov identifier NCT00863681) and CHEST-2 (ClinicalTrials.gov identifier NCT00910429) open-label extension studies in 
which all patients received riociguat $2.5 \mathrm{mg}$ three times daily maximum. Details of these trials are included in the supplementary material and published elsewhere $[23,24]$. The present analyses included patients who completed PATENT-1 or CHEST-1 and participated in PATENT-2 or CHEST-2.

The PATENT and CHEST studies were conducted in accordance with Good Clinical Practice guidelines and the Declaration of Helsinki. The institutional review board at each participating centre approved the study protocol and all patients gave written informed consent.

\section{Risk assessment}

Six of the 13 variables recommended for PAH risk stratification in the 2015 ESC/ERS treatment guidelines were collected prospectively in PATENT-1 and CHEST-1 (6MWD, WHO FC, NT-proBNP, RAP, cardiac index and $\mathrm{SvO}_{2}$ ). Using these variables, the aforementioned French invasive, French non-invasive and Swedish/COMPERA models were assessed post hoc in the PATENT and CHEST populations at baseline and follow-up. The resultant risk groups/strata were assessed for association with long-term outcomes (survival and clinical worsening-free survival).

Clinical worsening was prospectively defined as first occurrence of any of the following events: death, heart/lung transplantation, atrial septostomy in PATENT or rescue PEA due to worsening of pulmonary hypertension in CHEST, hospitalisation due to worsening of pulmonary hypertension, start of new specific pulmonary hypertension treatment, persistent decrease of $>15 \%$ from baseline in $6 \mathrm{MWD}$ (or $>30 \%$ compared with last study-related measurement) and persistent worsening of WHO FC. Independent adjudication of events was not undertaken.

\section{Statistical analysis}

Data from patients in the riociguat $2.5 \mathrm{mg}$ three times daily maximum group were included in all analyses detailed in this study. Data from patients in the riociguat $1.5 \mathrm{mg}$ three times daily maximum group in PATENT-1 were included in baseline demographics but excluded from all other analyses. Data from patients in the placebo group were included in baseline demographics and allocation of patients to risk group/strata, but excluded from any long-term assessments (Kaplan-Meier or Cox proportional hazards ratio analyses). All results are presented as observed, with no imputation for missing data.

Patients were grouped according to the number of low-risk criteria achieved (French registry methods) or assigned a risk stratum based on the rounded mean of risk grades (Swedish/COMPERA method) at baseline and follow-up in PATENT-1 and CHEST-1.

Kaplan-Meier analyses of observed data from patients in PATENT-1 and CHEST-1 were used to determine survival and clinical worsening-free survival in PATENT-2 and CHEST-2, respectively, according to the number of low-risk criteria achieved/risk stratum at baseline or follow-up of PATENT-1/ CHEST-1. Discrimination between Kaplan-Meier curves (prognostic groups) was determined using log-rank tests.

Cox proportional hazards models were performed in patients from PATENT-1 and CHEST-1 to assess the risk of death and clinical worsening over 2 years according to the number of low-risk criteria achieved/risk stratum (full details can be found in the supplementary material). Only patients who completed PATENT-1 and CHEST-1 and entered PATENT-2 and CHEST-2, respectively, were included in the current post hoc analyses.

Due to the small number of patients in the high-risk stratum using the Swedish/COMPERA model, patients in this group were excluded from all analyses evaluating statistical significance.

Throughout the text, the term "baseline" refers to PATENT-1 and CHEST-1 baseline, and "follow-up" refers to PATENT-1 week 12 or CHEST-1 week 16.

\section{Results}

PATENT studies of patients with PAH

Of the $443 \mathrm{PAH}$ patients included in PATENT-1, 396 participated in PATENT-2. In the PATENT-2 population at baseline, the majority of patients were female (80\%), white ethnicity (61\%), had idiopathic $\mathrm{PAH}(62 \%)$ and were in WHO FC III (54\%), with a mean \pm SD age of $50 \pm 16$ years. At entry into PATENT-1, $\sim 50 \%$ of the overall population were pre-treated with a PAH therapy and the mean time since diagnosis was 2.4 years (1.9 years in treatment-naive patients and 3.0 years in pre-treated patients), indicating a predominantly prevalent population. A total of 340 patients who participated in PATENT-2 were included in this analysis. Patient parameters according to baseline ESC/ERS risk stratification groups are shown in table 1. 
French registry invasive model

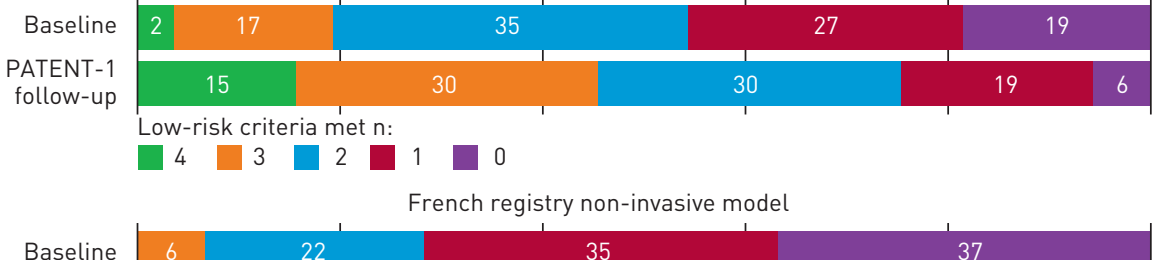

Baseline

PATENT-1

follow-up

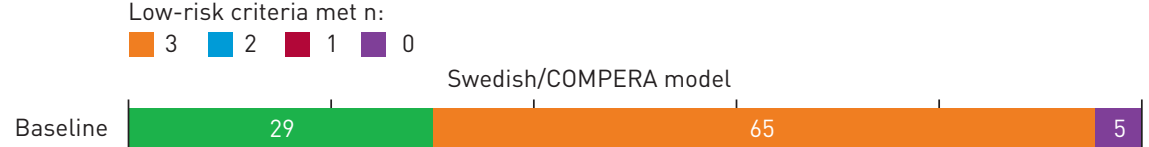

PATENT-1

follow-up

Low risk Intermediate risk High risk

French registry invasive model

\begin{tabular}{l|c|c|c|c|c|} 
c) & \multicolumn{5}{|c|}{ French registry invasive model } \\
$\begin{array}{r}\text { Baseline } \\
\text { CHEST-1 }\end{array}$ & 10 & 28 & 30 & \multicolumn{2}{|c|}{32} \\
follow-up & 15 & 20 & 32 & 24 & 9 \\
\hline
\end{tabular}

Low-risk criteria met $\mathrm{n}$ :

$4 \square$
$3 \quad 2 \square$

French registry non-invasive model

\begin{tabular}{|c|c|c|c|}
\hline Baseline & 2 & 29 & 54 \\
\hline $\begin{array}{l}\text { CHEST-1 } \\
\text { follow-up }\end{array}$ & 19 & 21 & 35 \\
\hline
\end{tabular}

Low-risk criteria met $\mathrm{n}$ :

$3 \square 2 \square 1 \square 0$
0

Swedish/COMPERA model

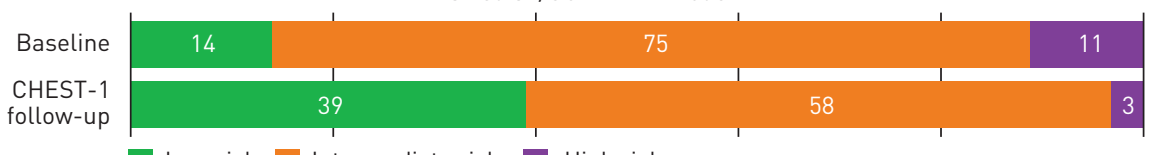

b)

\begin{tabular}{r|cccc|c|c|} 
Baseline & 4 & 17 & & 39 & 28 & 13 \\
\cline { 2 - 6 } & & & 39 & 30 & 16 \\
\hline $\begin{array}{r}\text { PATENT-1 } \\
\text { follow-up }\end{array}$ & 9 & 20 & 24 & &
\end{tabular}

Low-risk criteria met $\mathrm{n}$ :

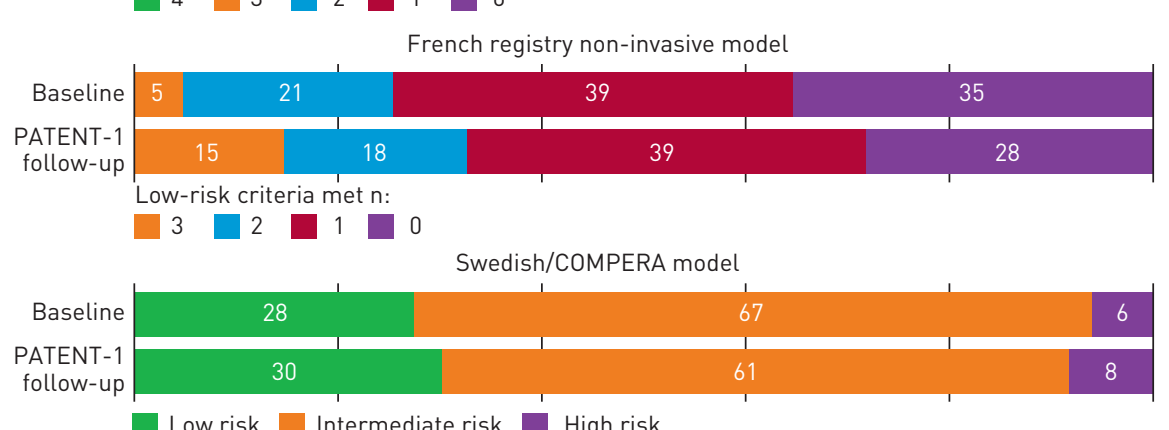

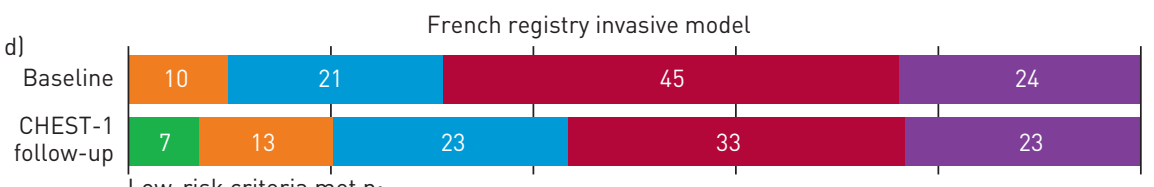

Low-risk criteria met $\mathrm{n}$ :

\section{\begin{tabular}{ll|l}
4 & 3 & 2 \\
1 & 0
\end{tabular}}

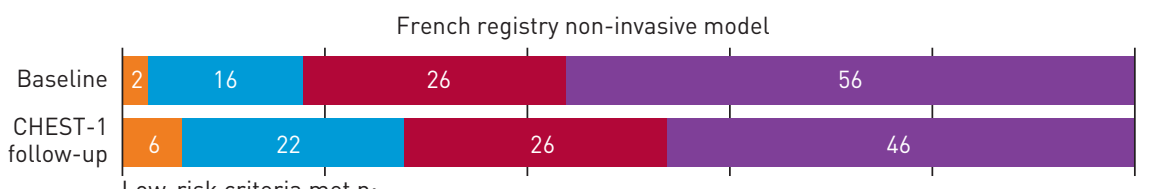

Low-risk criteria met $\mathrm{n}$ :

\section{\begin{tabular}{l}
\hline \\
\hline
\end{tabular} $2 \square 1 \square 0$}

\begin{tabular}{|c|c|c|c|}
\hline \multicolumn{4}{|c|}{ Swedish/COMPERA model } \\
\hline Baseline & 9 & 82 & 10 \\
\hline $\begin{array}{l}\text { CHEST-1 } \\
\text { follow-up }\end{array}$ & 21 & 65 & 15 \\
\hline
\end{tabular}

FIGURE 1 Proportion of patients in each risk stratum at baseline and follow-up receiving al riociguat and b) placebo in PATENT-1, and receiving cl riociguat and d) placebo in CHEST-1, according to the three abbreviated versions of the European Society of Cardiology (ESC)/European Respiratory Society (ERS) risk stratification model. Data may not add up to $100 \%$ due to rounding. Only patients who participated in PATENT-2 and CHEST-2 were included in the analysis. The analysis is based on observed data with no imputation. French registry invasive rounding. Only patients who participated in PATENT-2 and CHEST-2 were included in the analysis. The analysis is based on observed data with no imputation. French registry invasive cardiac index $\geqslant 2.5 \mathrm{~L} \cdot \mathrm{min}^{-1} \cdot \mathrm{m}^{-2}$. French registry non-invasive method: number of low-risk criteria fulfilled: $6 \mathrm{MWD}>440 \mathrm{~m}$, WHO FC I/II and N-terminal pro-brain natriuretic peptide (NT-proBNP) $<300 \mathrm{pg} \cdot \mathrm{mL}^{-1}$. Swedish/COMPERA method: mean of grades (1-3: low, intermediate, high) of six available criteria (6MWD, WHO FC, NT-proBNP, RAP, cardiac index and mixed venous oxygen saturation) as defined in the ESC/ERS 2015 treatment guidelines, rounded to the nearest integer. 


\section{French registry invasive model}

4 criteria versus 0 criteria

3 criteria versus 0 criteria

2 criteria versus 0 criteria

1 criterion versus 0 criteria

French registry non-invasive mode

3 criteria versus 0 criteri

2 criteria versus 0 criteria

1 criterion versus 0 criteria

Swedish/COMPERA model\#

Low risk versus

intermediate risk

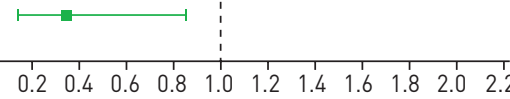

Hazard ratio $(95 \% \mathrm{CI})$

French registry invasive model

4 criteria versus 0 criteria

3 criteria versus 0 criteria

2 criteria versus 0 criteria

1 criterion versus 0 criteria

French registry non-invasive mode

3 criteria versus 0 criteria

2 criteria versus 0 criteria

1 criterion versus 0 criteria

Swedish/COMPERA model ${ }^{\#}$

Low risk versus

intermediate risk

Hazard ratio
$(95 \% \mathrm{Cl})$

$0.439(0.205-0.939) \quad 0.034$

$0.769(0.438-1.349) \quad 0.359$

$0.657(0.356-1.213) \quad 0.180$

$0.118(0.016-0.859) \quad 0.035$

$0.461(0.244-0.871) \quad 0.017$

$0.702(0.436-1.131) \quad 0.146$

$0.564(0.324-0.980) \quad 0.042$ b)

French registry invasive model

4 criteria versus 0 criteria

3 criteria versus 0 criteria

2 criteria versus 0 criteria

1 criterion versus 0 criteria

French registry non-invasive model

3 criteria versus 0 criteria

2 criteria versus 0 criteria

1 criterion versus 0 criteria

Swedish/COMPERA model ${ }^{\#}$

Low risk versus

intermediate risk

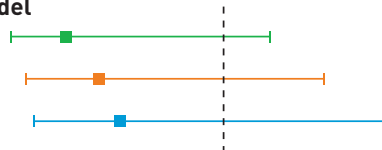

$195 \% \mathrm{Cl})$

$0.324(0.087-1.208) \quad 0.093$

$0.462(0.149-1.433) \quad 0.181$

$0.554(0.180-1.701) \quad 0.302$

$0.691(0.211-2.263) \quad 0.541$

$0.178(0.059-0.536) \quad 0.002$

$0.457(0.219-0.954) \quad 0.037$

$0.447(0.207-0.963) \quad 0.040$

$0.409(0.215-0.779) \quad 0.007$

\begin{tabular}{llllll}
\hline & 1 & & & & \\
0.0 & 0.5 & 1.0 & 1.5 & 2.0 & 2.5
\end{tabular}

d)

French registry invasive model

4 criteria versus 0 criteria

3 criteria versus 0 criteria

2 criteria versus 0 criteria

1 criterion versus 0 criteria

r.

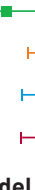

French registry non-invasive mode

3 criteria versus 0 criteria

2 criteria versus 0 criteria

1 criterion versus 0 criteria

Swedish/COMPERA model ${ }^{\#}$

Low risk versus

intermediate risk

Hazard ratio p-value $195 \% \mathrm{Cl})$

$0.152(0.053-0.44) \quad 0.001$

$0.427(0.193-0.943) \quad 0.035$

$0.403(0.181-0.897) \quad 0.026$

$0.426(0.179-1.015) \quad 0.054$

$0.189(0.082-0.436) 9.5 \times 10^{-1}$

$0.610(0.355-1.047) \quad 0.073$

$0.531(0.301-0.938) \quad 0.029$

$0.541(0.344-0.851) \quad 0.008$

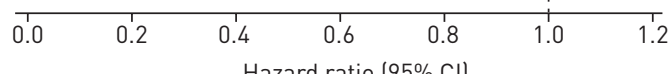

FIGURE 2 Forest plots comparing survival in PATENT-2 expressed as hazard ratios (95\% CI) for mortality comparing patients who achieved one or more low-risk criteria compared with those who achieved no low-risk criteria at al baseline and b) follow-up in PATENT-1, and clinical worsening-free survival in PATENT-2 expressed as hazard ratios (95\% CI) for clinical worsening comparing patients who achieved one or more low-risk criteria compared with those who achieved no low-risk criteria at c) baseline and d) follow-up in PATENT-1. Only patients receiving riociguat $2.5 \mathrm{mg}$ three times daily maximum in PATENT-1 who participated in PATENT-2 were considered in this analysis. French registry invasive method: number of low-risk criteria fulfilled: 6 - min walk distance (6MWD) $>440 \mathrm{~m}$. World Health Organization (WHO) functional class (FC) I/II, right atrial pressure (RAP) $<8 \mathrm{mmHg}$ and cardiac index $\geqslant 2.5 \mathrm{~L} \cdot \mathrm{min}^{-1} \cdot \mathrm{m}^{-2}$. French registry non-invasive method: number of low-risk criteria fulfilled: $6 \mathrm{MWD}>440 \mathrm{~m}$, WHO FC I/II and N-terminal pro-brain natriuretic peptide (NT-proBNP) $<300$ pg.mL $\mathrm{mL}^{-1}$. Swedish/ COMPERA method: mean of grades (1-3: low, intermediate, high) of six available criteria (6MWD, WHO FC, NT-proBNP, RAP, cardiac index and mixed venous oxygen saturation) as defined in the European Society of Cardiology/European Respiratory Society 2015 treatment guidelines, rounded to the nearest integer. ${ }^{\#}$ : due to the small number of patients in the high-risk category at baseline and follow-up according to the Swedish/COMPERA method, these patients were not included in this analysis. Due to no or few events in some risk groups/strata, hazards ratios could not be calculated in some cases. 


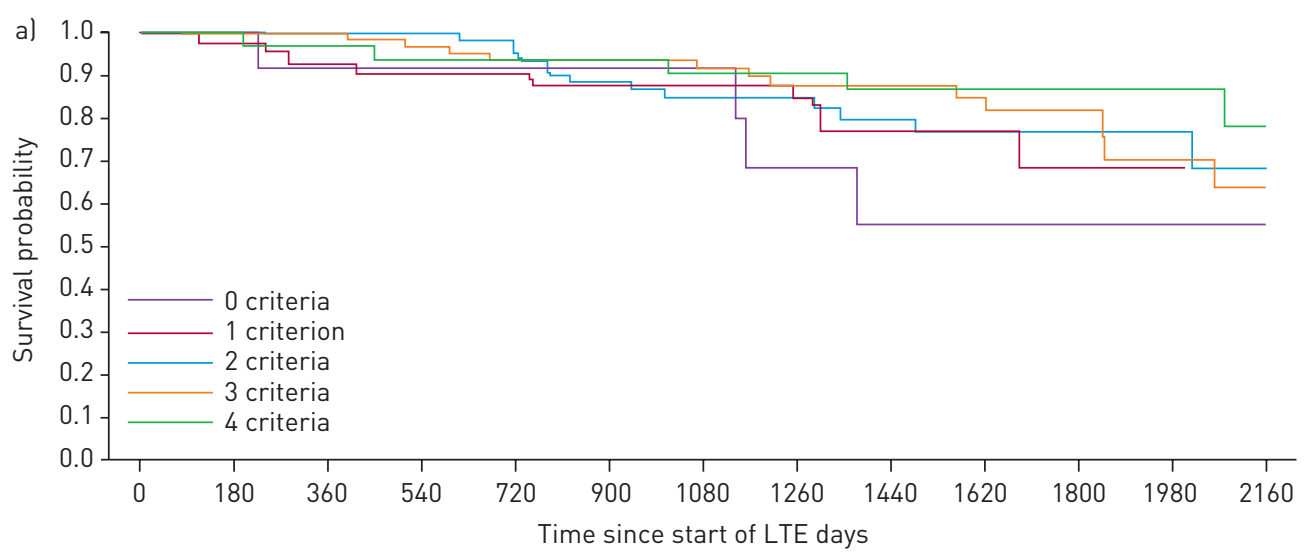

Patients at risk $\mathrm{n}$ :

$\begin{array}{ccccccccccccc}-13 & 12 & 9 & 9 & 8 & 8 & 8 & 6 & 4 & 4 & 3 & 3 & 1 \\ -44 & 41 & 36 & 35 & 35 & 31 & 29 & 25 & 14 & 10 & 3 & 1 & 0 \\ -70 & 66 & 65 & 61 & 58 & 51 & 46 & 35 & 30 & 20 & 15 & 10 & 5 \\ -69 & 66 & 66 & 63 & 59 & 56 & 49 & 41 & 34 & 28 & 16 & 11 & 7 \\ -34 & 33 & 31 & 30 & 29 & 29 & 28 & 24 & 22 & 18 & 13 & 11 & 9\end{array}$

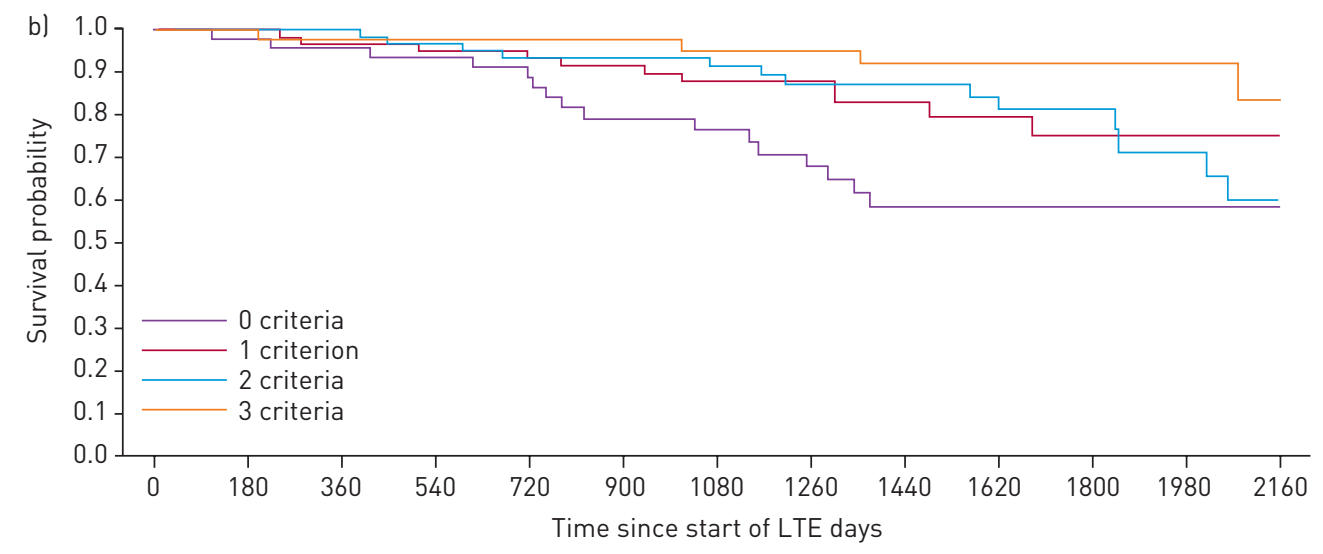

Patients at risk $\mathrm{n}$ :

$\begin{array}{rllllllllllll}-51 & 48 & 43 & 41 & 38 & 30 & 28 & 23 & 16 & 10 & 8 & 4 & 2 \\ -67 & 63 & 59 & 56 & 55 & 51 & 46 & 36 & 26 & 20 & 10 & 7 & 3 \\ -67 & 64 & 64 & 60 & 55 & 54 & 48 & 40 & 33 & 28 & 17 & 13 & 7 \\ -46 & 44 & 42 & 42 & 42 & 41 & 38 & 32 & 29 & 22 & 15 & 12 & 10\end{array}$

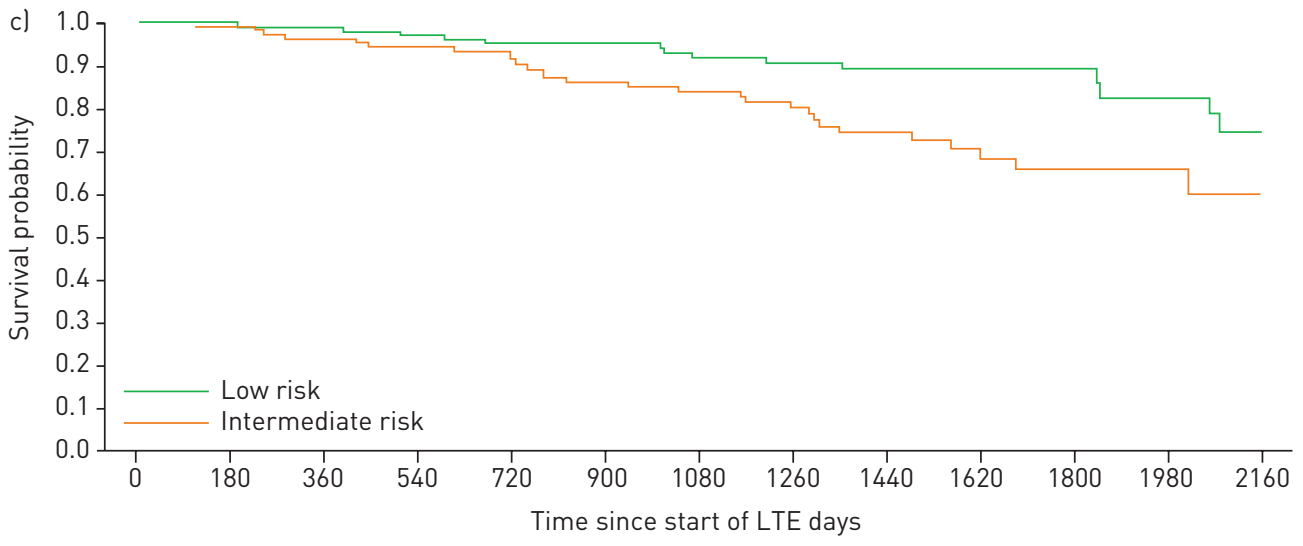

Patients at risk $\mathrm{n}$ :

$\begin{array}{ccccccccccccc}-113 & 107 & 105 & 101 & 97 & 94 & 84 & 69 & 60 & 49 & 31 & 23 & 16 \\ -115 & 109 & 100 & 95 & 90 & 79 & 73 & 60 & 44 & 31 & 19 & 13 & 6\end{array}$


FIGURE 3 Kaplan-Meier curves for survival in patients based on risk stratification at PATENT-1 follow-up: a) French registry invasive method, b) French registry non-invasive method and c) Swedish/COMPERA method. Only patients receiving riociguat $2.5 \mathrm{mg}$ three times daily maximum in PATENT-1 who participated in PATENT-2 were considered in this analysis. Data were based on observed cases with no imputation. Day $0=$ start of extension study. Only three patients were in the Swedish/COMPERA high-risk group at PATENT-1 follow-up and were therefore not included in the analysis for this method. French registry invasive method: number of low-risk criteria fulfilled: 6-min walk distance (6MWD) $>440 \mathrm{~m}$, World Health Organization (WHO) functional class (FC) I/II, right atrial pressure (RAP) $<8 \mathrm{mmHg}$ and cardiac index $\geqslant 2.5 \mathrm{~L} \cdot \mathrm{min}^{-1} \cdot \mathrm{m}^{-2}$. French registry non-invasive method: number of low-risk criteria fulfilled: 6MWD $>440 \mathrm{~m}$, WHO FC I/II and $\mathrm{N}$-terminal pro-brain natriuretic peptide (NT-proBNP) $<300 \mathrm{pg} \cdot \mathrm{mL}^{-1}$. Swedish/COMPERA method: mean of grades (1-3: low, intermediate, high) of six available criteria (6MWD, WHO FC, NT-proBNP, RAP, cardiac index and mixed venous oxygen saturation) as defined in the European Society of Cardiology/European Respiratory Society 2015 treatment guidelines, rounded to the nearest integer. Log-rank test: invasive $p=0.5595$, noninvasive $p=0.0126$ and Swedish/COMPERA $p=0.0099$. LTE: long-term extension.

Assessment of the patient demographics in each risk group/strata at baseline and follow-up showed no difference between patients in each group/strata, and thus no obvious confounding factors that may have influenced long-term outcomes could be identified (data not shown).

Risk stratification at baseline and association with long-term outcomes

Risk stratification at baseline according to the three abbreviated methods is summarised in figure $1 \mathrm{a}$ and $\mathrm{b}$. Using the French non-invasive method, when stratified by number of low-risk criteria achieved at

TABLE 1 European Society of Cardiology/European Respiratory Society risk assessment parameters in the PATENT-2 study population at baseline and follow-up of PATENT-1

\begin{tabular}{|c|c|c|c|c|}
\hline & \multicolumn{2}{|c|}{ Riociguat $^{\#}$} & \multicolumn{2}{|c|}{ Placebo } \\
\hline & Baseline & Follow-up & Baseline & Follow-up \\
\hline Subjects & \multicolumn{2}{|c|}{231} & \multicolumn{2}{|c|}{109} \\
\hline 6MWD risk group & & & & \\
\hline Missing & 0 & $3(1)$ & 0 & $1(1)$ \\
\hline Low: >440 m & $26(11)$ & 75 (32) & $15(14)$ & $32(29)$ \\
\hline Intermediate: $165-440 \mathrm{~m}$ & 202 (87) & $152(66)$ & $94(86)$ & $76(70)$ \\
\hline High: $<165 \mathrm{~m}$ & $3(1)$ & $1(<1)$ & 0 & 0 \\
\hline \multicolumn{5}{|l|}{ WHO FC risk group } \\
\hline Missing & 0 & $3(1)$ & $1(1)$ & $1(1)$ \\
\hline Low: I/II & $103(45)$ & $142(61)$ & $57(52)$ & $62(57)$ \\
\hline Intermediate: III & $128(55)$ & 85 (37) & $49(45)$ & $44(40)$ \\
\hline High: IV & 0 & $1(<1)$ & $2(2)$ & $2(2)$ \\
\hline \multicolumn{5}{|l|}{ NT-proBNP risk group } \\
\hline Missing & $21(9)$ & $4(2)$ & $12(11)$ & $3(3)$ \\
\hline Low: $<300 \mathrm{pg} \cdot \mathrm{mL}^{-1}$ & $94(41)$ & $122(53)$ & $32(29)$ & $36(33)$ \\
\hline Intermediate: $300-1400 \mathrm{pg} \cdot \mathrm{mL}^{-1}$ & $65(28)$ & $82(35)$ & $39(36)$ & $36(33)$ \\
\hline High: $>1400 \mathrm{pg} \cdot \mathrm{mL}^{-1}$ & $51(22)$ & $23(10)$ & $26(24)$ & 34 (31) \\
\hline \multicolumn{5}{|l|}{ RAP risk group } \\
\hline Missing & $1(<1)$ & $2(1)$ & $5(5)$ & $6(6)$ \\
\hline Low: $<8 \mathrm{mmHg}$ & $131(57)$ & $138(60)$ & $62(57)$ & $51(47)$ \\
\hline Intermediate: $8-14 \mathrm{mmHg}$ & $72(31)$ & $74(32)$ & $37(34)$ & $39(36)$ \\
\hline High: $>14 \mathrm{mmHg}$ & $27(12)$ & $17(7)$ & $5(5)$ & $13(12)$ \\
\hline \multicolumn{5}{|l|}{ Cardiac index risk group } \\
\hline Missing & $3(1)$ & $4(2)$ & $5(5)$ & $6(6)$ \\
\hline Low: $\geqslant 2.5 \mathrm{~L} \cdot \mathrm{min}^{-1} \cdot \mathrm{m}^{-2}$ & $102(44)$ & $172(74)$ & $52(48)$ & $46(42)$ \\
\hline Intermediate: $2.0-2.5 \mathrm{~L} \cdot \mathrm{min}^{-1} \cdot \mathrm{m}^{-2}$ & $83(36)$ & $40(17)$ & $21(19)$ & $30(28)$ \\
\hline High: $<2.0 \mathrm{~L} \cdot \mathrm{min}^{-1} \cdot \mathrm{m}^{-2}$ & $43(19)$ & $15(6)$ & $31(28)$ & $27(25)$ \\
\hline \multicolumn{5}{|l|}{$\mathrm{SvO}_{2}$ risk group } \\
\hline Missing & $26(11)$ & $11(5)$ & $12(11)$ & $11(10)$ \\
\hline Low: $>65 \%$ & $106(46)$ & $154(67)$ & $56(51)$ & $47(43)$ \\
\hline Intermediate: $60-65 \%$ & $46(20)$ & $36(16)$ & $26(24)$ & $25(23)$ \\
\hline High: $<60 \%$ & $53(23)$ & 30 (13) & $15(14)$ & $26(24)$ \\
\hline
\end{tabular}

Data are presented as $\mathrm{n}$ or $\mathrm{n}$ (\%). 6MWD: 6-min walk distance; WHO: World Health Organization; FC: functional class; NT-proBNP: N-terminal pro-brain natriuretic peptide; RAP: right atrial pressure; $\mathrm{SvO}_{2}$ : mixed venous oxygen saturation. ${ }^{\#}$ : riociguat $2.5 \mathrm{mg}$ three times daily maximum. 


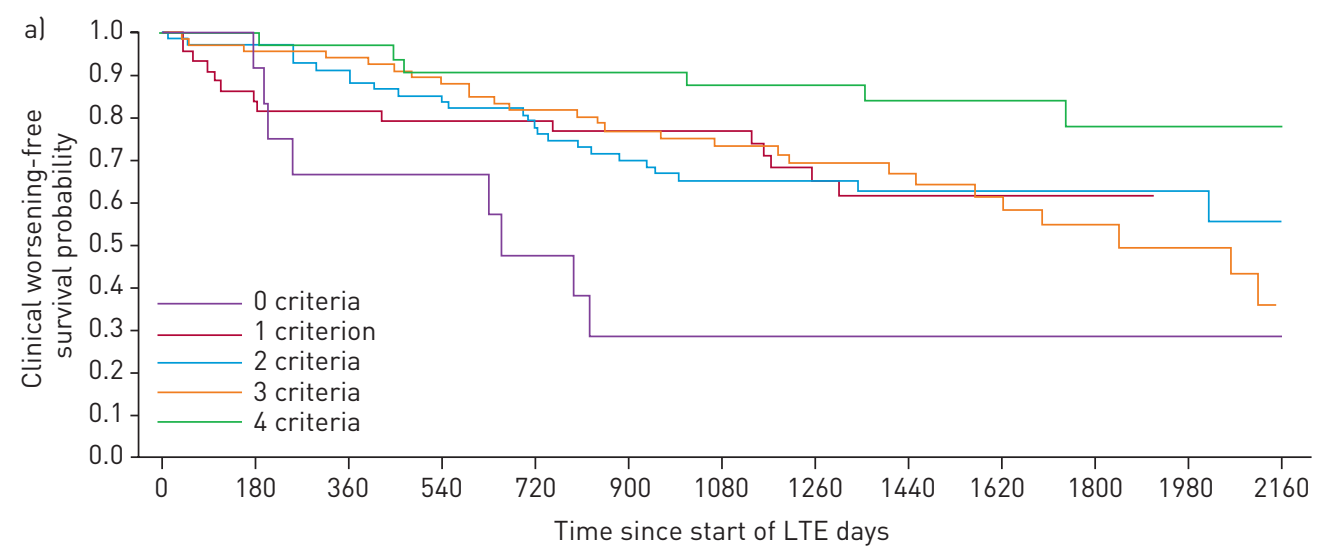

Patients at risk $\mathrm{n}$ :

$\begin{array}{ccccccccccccc}-13 & 11 & 7 & 7 & 5 & 3 & 3 & 3 & 3 & 3 & 2 & 2 & 1 \\ -44 & 36 & 34 & 33 & 33 & 29 & 27 & 20 & 10 & 7 & 2 & 0 & 0 \\ -70 & 66 & 61 & 55 & 51 & 44 & 39 & 28 & 25 & 18 & 14 & 10 & 5 \\ -69 & 63 & 62 & 58 & 52 & 46 & 40 & 34 & 26 & 20 & 11 & 8 & 5 \\ -34 & 33 & 31 & 29 & 29 & 29 & 28 & 24 & 22 & 18 & 13 & 10 & 9\end{array}$

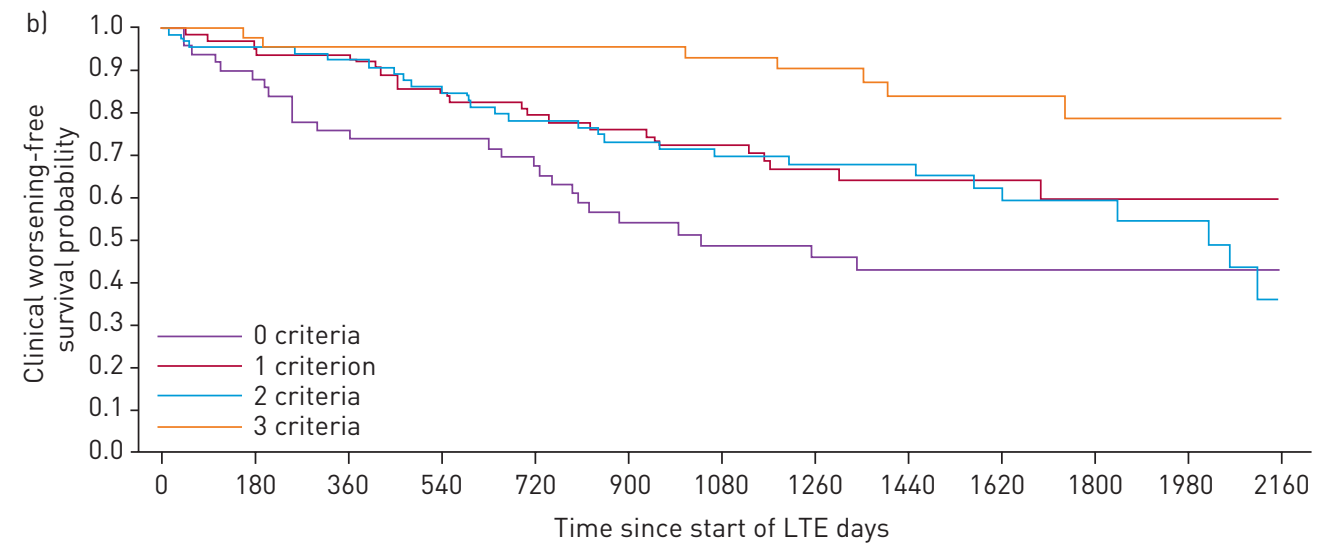

Patients at risk $\mathrm{n}$ :

$\begin{array}{ccccccccccccc}-51 & 44 & 36 & 35 & 32 & 22 & 19 & 16 & 12 & 8 & 6 & 3 & 2 \\ -67 & 61 & 59 & 52 & 49 & 45 & 41 & 29 & 20 & 16 & 9 & 6 & 3 \\ -67 & 62 & 60 & 55 & 49 & 45 & 40 & 32 & 27 & 21 & 12 & 10 & 5 \\ -46 & 43 & 41 & 41 & 41 & 40 & 37 & 32 & 27 & 21 & 15 & 11 & 10\end{array}$

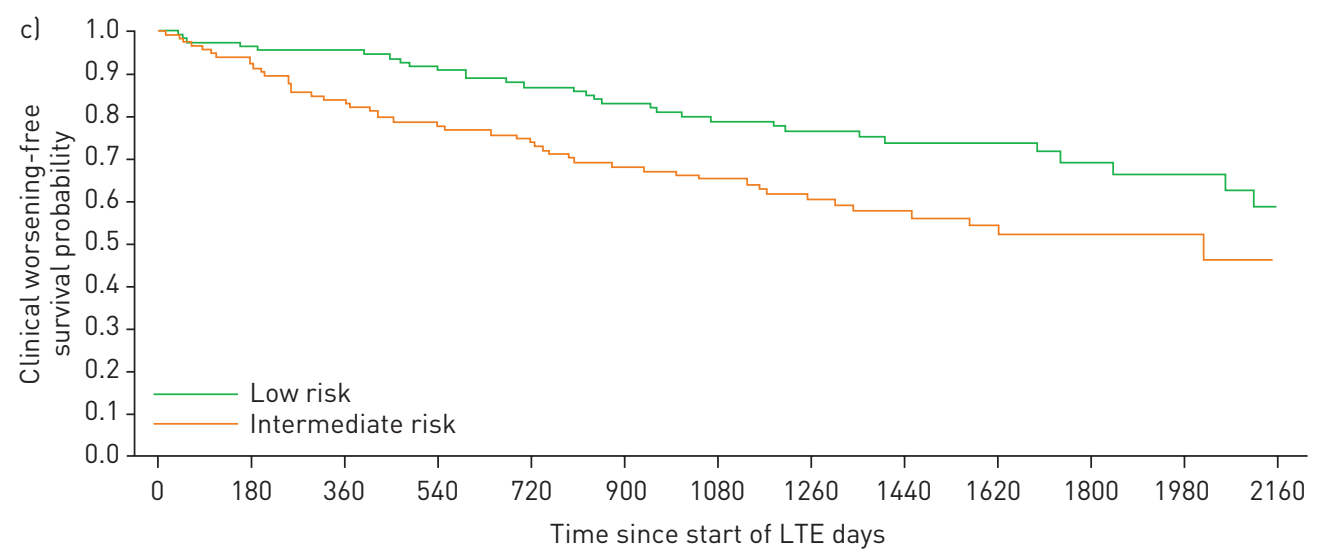

Patients at risk $\mathrm{n}$ :

$\begin{array}{lllllllllllll}-113 & 104 & 102 & 96 & 90 & 83 & 75 & 61 & 51 & 41 & 27 & 20 & 15 \\ -115 & 103 & 91 & 84 & 80 & 69 & 62 & 48 & 35 & 25 & 15 & 10 & 5\end{array}$


FIGURE 4 Kaplan-Meier curves for clinical worsening-free survival in patients based on risk stratification at PATENT-1 follow-up: a) French registry invasive method, b) French registry non-invasive method and c) Swedish/COMPERA method. Only patients receiving riociguat $2.5 \mathrm{mg}$ three times daily maximum in PATENT-1 who participated in PATENT-2 were considered in this analysis. Data were based on observed cases with no imputation. Day $0=$ start of extension study. Only three patients were in the Swedish/COMPERA high-risk group at PATENT-1 follow-up and were therefore not included in the analysis for this method. French registry invasive method: number of low-risk criteria fulfilled: 6-min walk distance (6MWD) $>440 \mathrm{~m}$, World Health Organization (WHO) functional class (FC) $1 /$ II, right atrial pressure (RAP) $<8 \mathrm{mmHg}$ and cardiac index $\geqslant 2.5 \mathrm{~L} \cdot \mathrm{min}^{-1} \cdot \mathrm{m}^{-2}$. French registry non-invasive method: number of low-risk criteria fulfilled: 6MWD $>440 \mathrm{~m}$, WHO FC I/II and N-terminal pro-brain natriuretic peptide (NT-proBNP) $<300 \mathrm{pg} \cdot \mathrm{mL}^{-1}$. Swedish/ COMPERA method: mean of grades (1-3: low, intermediate, high) of six available criteria (6MWD, WHO FC, NT-proBNP, RAP, cardiac index and mixed venous oxygen saturation) as defined in the European Society of Cardiology/European Respiratory Society 2015 treatment guidelines, rounded to the nearest integer. Log-rank test: invasive $p=0.0078$, non-invasive $p=0.0001$ and Swedish/COMPERA $p=0.0023$. LTE: long-term extension.

PATENT-1 baseline, there was a significant difference between prognostic groups for both survival $(\mathrm{p}=0.0283)$ and clinical worsening-free survival $(\mathrm{p}=0.0036)$, whereas the French invasive method did not discriminate between prognostic groups for either outcome (supplementary figures E1 and E2). Discrimination between prognostic groups for both survival $(\mathrm{p}=0.0355)$ and clinical worsening-free survival $(\mathrm{p}=0.0236$ ) based on baseline risk strata was also observed with the Swedish/COMPERA method (supplementary figures E1 and E2).

Cox proportional hazards analyses of the risk of death or clinical worsening in PATENT-2 according to the number of low-risk criteria/risk strata at baseline are shown in figure $2 \mathrm{a}$ and $\mathrm{c}$. The French invasive and non-invasive methods showed lower risks of death or clinical worsening as more low-risk criteria were present at baseline. Compared with achieving no low-risk criteria, these reductions were statistically significant for clinical worsening in patients who met three criteria with the invasive strategy or two or more criteria with the non-invasive strategy. Using the Swedish/COMPERA approach, patients categorised as low risk at baseline had a $64 \%$ lower risk of death and a $44 \%$ lower risk of a clinical worsening event over 2 years than patients in the intermediate-risk group.

\section{Risk stratification at follow-up and association with long-term outcomes}

In PATENT-1, treatment with riociguat increased the number of low-risk criteria achieved (French invasive and non-invasive methods) and improved risk strata using the Swedish/COMPERA method at follow-up (figure 1a and $b$ ).

When patients were stratified by the number of low-risk criteria achieved at PATENT-1 follow-up, the French non-invasive method was able to discriminate between prognostic groups for both survival ( $\mathrm{p}=0.0126)$ and clinical worsening-free survival $(\mathrm{p}=0.0001)$, whereas the invasive method only discriminated for clinical worsening-free survival $(\mathrm{p}=0.0078)$. Based on risk strata at follow-up, the Swedish/COMPERA method discriminated between prognostic groups for both survival $(\mathrm{p}=0.0099)$ and clinical worsening-free survival $(\mathrm{p}=0.0023)$ (figures 3 and 4$)$. Estimated survival rates over 3 years according to the number of low-risk criteria achieved or risk strata achieved at follow-up are shown in supplementary table E1.

For the French invasive method, achieving two or more low-risk criteria at PATENT-1 follow-up was associated with a significantly lower risk of clinical worsening compared with achieving none (figure $2 \mathrm{~d}$ ). Using the French non-invasive method, achieving one or more low-risk criteria at follow-up was associated with a significant reduction in the risk of death. Similar results were seen for clinical worsening (although no significant difference in clinical worsening was seen when patients achieving two low-risk criteria were compared with those achieving none). Using the Swedish/COMPERA method, patients categorised as low risk at PATENT-1 follow-up had a 59\% lower risk of death and a $46 \%$ lower risk of a clinical worsening event than patients in the intermediate-risk group (figure $2 \mathrm{~b}$ and $\mathrm{d}$ ).

\section{CHEST studies of patients with inoperable or persistent/recurrent CTEPH}

Of the 261 CTEPH patients included in CHEST-1, 237 participated in CHEST-2 and were included in the analyses. At CHEST-1 baseline, the majority of patients were female (65\%), white ethnicity (70\%) and were in WHO FC III (65\%), with a mean \pm SD age of $59 \pm 13$ years; $73 \%$ were inoperable and $27 \%$ had persistent/recurrent CTEPH. At entry into CHEST-1, the mean \pm sD time since diagnosis was $2.1 \pm 3.1$ years and the time since operation in the post-operative patients $(n=65)$ was $3.3 \pm 3.0$ years.

With the abbreviated methods, patients receiving riociguat in CHEST-1 met more low-risk criteria or improved their risk strata at follow-up compared with baseline (figure 1c and d). Risk stratification using 


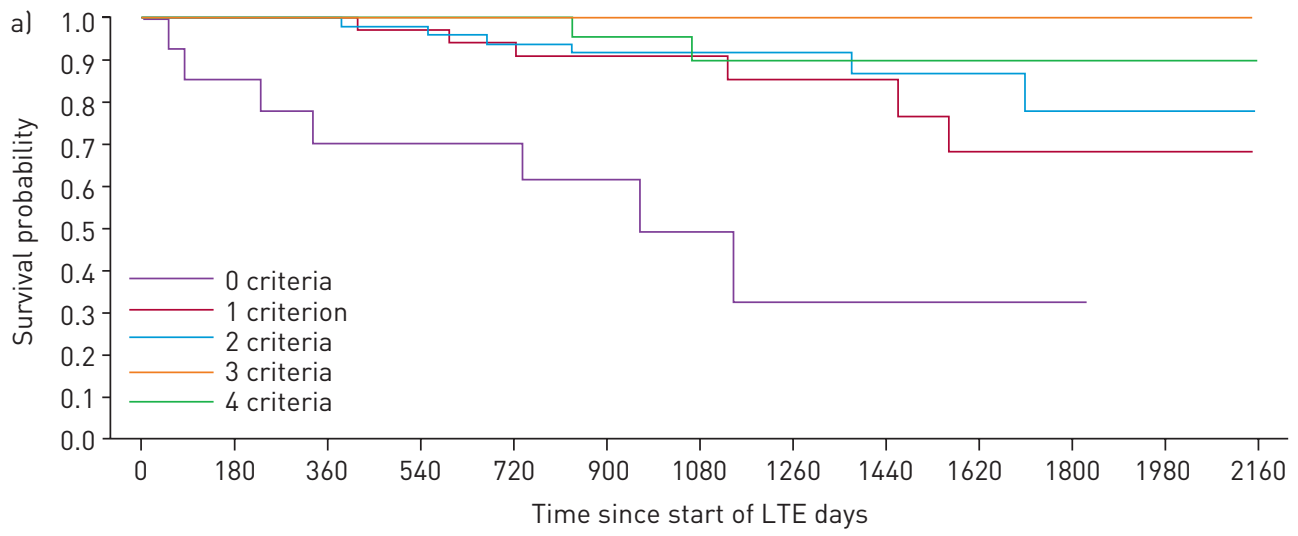

Patients at risk $\mathrm{n}$ :

$\begin{array}{ccccccccccccc}-14 & 11 & 9 & 8 & 8 & 6 & 3 & 2 & 2 & 1 & 1 & 0 & 0 \\ -37 & 36 & 34 & 33 & 31 & 22 & 17 & 13 & 10 & 8 & 6 & 1 & 1 \\ -50 & 50 & 50 & 48 & 45 & 38 & 30 & 23 & 15 & 13 & 8 & 4 & 2 \\ -31 & 30 & 29 & 29 & 28 & 26 & 22 & 20 & 15 & 12 & 7 & 2 & 1 \\ -23 & 23 & 23 & 23 & 23 & 21 & 16 & 14 & 11 & 10 & 7 & 4 & 3\end{array}$

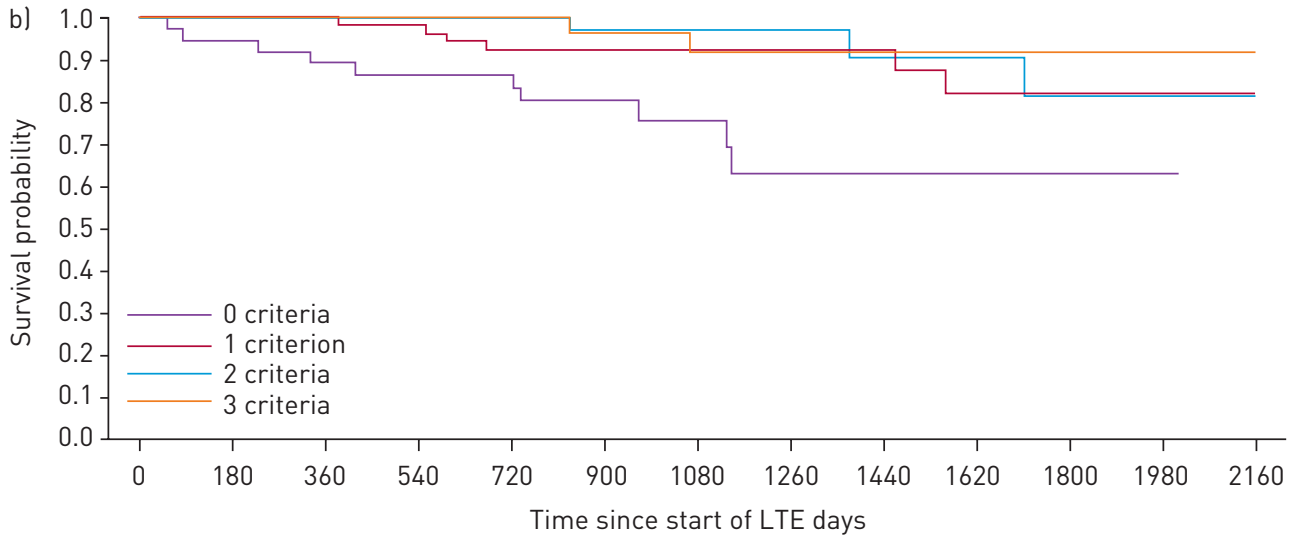

Patients at risk $\mathrm{n}$ :

\begin{tabular}{|c|c|c|c|c|c|c|c|c|c|c|c|}
\hline-37 & 34 & 32 & 30 & 29 & 22 & 13 & 9 & 8 & 6 & 4 & 1 \\
\hline-55 & 54 & 52 & 50 & 46 & 38 & 31 & 25 & 19 & 15 & 11 & 5 \\
\hline-33 & 32 & 31 & 31 & 31 & 27 & 24 & 19 & 13 & 12 & 8 & 2 \\
\hline-30 & 30 & 30 & 30 & 29 & 26 & 20 & 19 & 13 & 11 & 6 & 3 \\
\hline
\end{tabular}

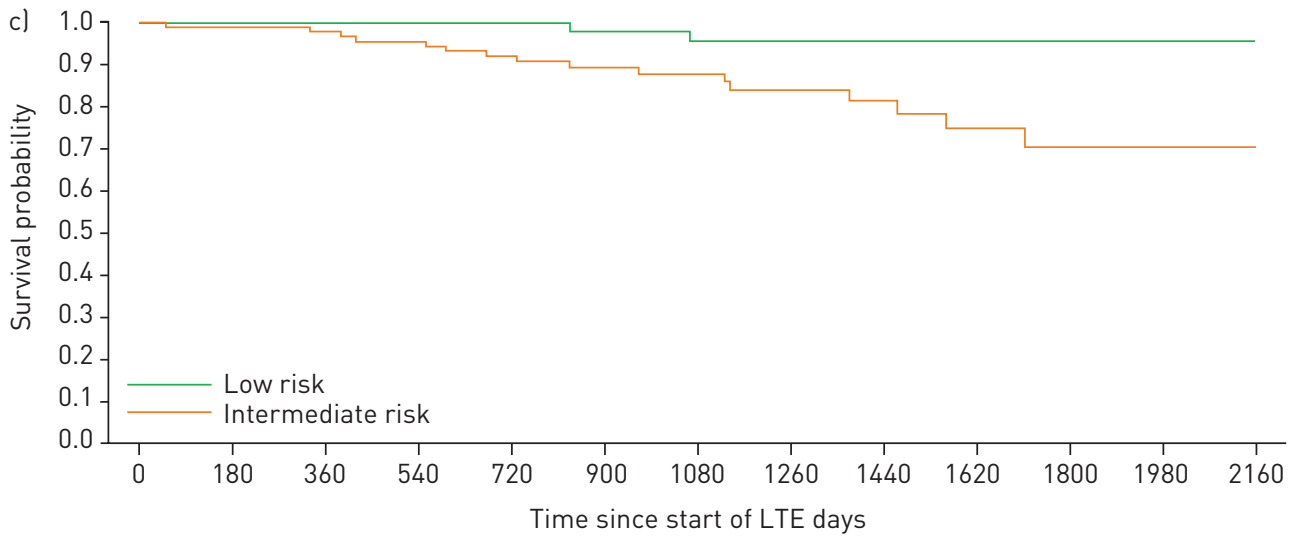

Patients at risk $\mathrm{n}$ :

$\begin{array}{rllllllllllll}-60 & 60 & 59 & 58 & 58 & 52 & 39 & 34 & 26 & 22 & 14 & 5 & 4 \\ -90 & 87 & 84 & 82 & 76 & 61 & 49 & 38 & 27 & 22 & 15 & 6 & 3\end{array}$


FIGURE 5 Kaplan-Meier curves for survival in patients based on risk stratification at CHEST-1 follow-up: a) French registry invasive method, b) French registry non-invasive method and c) Swedish/COMPERA method. Only patients receiving riociguat $2.5 \mathrm{mg}$ three times daily maximum in CHEST-1 who participated in CHEST-2 were considered in this analysis. Data were based on observed cases with no imputation. Day $0=$ start of extension study. Only five patients were in the Swedish/COMPERA high-risk group at CHEST-1 follow-up and were therefore not included in the analysis for this method. French registry invasive method: number of low-risk criteria fulfilled: 6-min walk distance (6MWD) $>440 \mathrm{~m}$, World Health Organization (WHO) functional class (FC) I/II, right atrial pressure (RAP) $<8 \mathrm{mmHg}$ and cardiac index $\geqslant 2.5 \mathrm{~L} \cdot \mathrm{min}^{-1} \cdot \mathrm{m}^{-2}$. French registry non-invasive method: number of low-risk criteria fulfilled: $6 \mathrm{MWD}>440 \mathrm{~m}$, WHO FC $\mathrm{I} / \mathrm{II}$ and $\mathrm{N}$-terminal pro-brain natriuretic peptide (NT-proBNP) $<300 \mathrm{pg} \cdot \mathrm{mL}^{-1}$. Swedish/COMPERA method: mean of grades (1-3: low, intermediate, high) of six available criteria (6MWD, WHO FC, NT-proBNP, RAP, cardiac index and mixed venous oxygen saturation) as defined in the European Society of Cardiology/European Respiratory Society 2015 treatment guidelines, rounded to the nearest integer. Log-rank test: invasive $p<0.0001$, noninvasive $p=0.0231$ and Swedish/COMPERA $p<0.0001$. LTE: long-term extension.

all three abbreviated models at CHEST-1 follow-up discriminated prognostic groups for both survival and clinical worsening-free survival in CHEST-2 (figures 5 and 6).

The risk of death and clinical worsening in CHEST-2 according to the number of low-risk criteria or risk strata at follow-up (Cox proportional hazards analyses) is summarised in figure 7. According to both French registry methods, achieving one or more low-risk criteria was associated with a reduction in both the risk of death and clinical worsening over 2 years compared with achieving no low-risk criteria. For the Swedish/COMPERA method, patients stratified as low risk at week 16 had a decreased risk of death or clinical worsening compared with those in the intermediate-risk stratum.

\section{Discussion}

Current ESC/ERS pulmonary hypertension treatment guidelines recommend a goal-oriented treatment strategy, with periodic assessment of patients using a risk stratification model with a number of modifiable parameters [1, 12]. Retrospective studies from three registries (French, Swedish and COMPERA), collectively evaluating over 3000 patients with incident PAH, assessed abbreviated methods of the ESC/ ERS risk stratification model. These registry analyses have consistently highlighted the favourable prognostic outcome of "low-risk" versus other risk categories [13-15]. While these observational studies support a goal-oriented treatment strategy with a low-risk profile as the overall objective, such a strategy has not yet been validated prospectively, in a clinical trial setting or in a prevalent PAH population [29]. The current post hoc analysis is the first to assess these abbreviated methods in a mostly prevalent population of patients with PAH from a pivotal clinical study.

Analysis of patients with PAH enrolled in the PATENT studies found that only a small number of patients achieved all of the assessed ESC/ERS low-risk criteria at PATENT-1 follow-up [30]. However, 12 weeks of riociguat treatment increased the number of patients achieving a greater number of low-risk criteria (French methods) or a low-risk stratum (Swedish/COMPERA method) compared with patients receiving placebo.

Assessment of long-term outcomes of patients with PAH grouped according to the number of low-risk criteria or risk strata found that the French non-invasive and the Swedish/COMPERA methods discriminated prognosis for both survival and clinical worsening-free survival over 2 years in the PATENT-2 study. Patients achieving one or more low-risk non-invasive criteria at follow-up had a significant reduction in the risk of death, based on Cox proportional hazards ratios. Similar results were seen for clinical worsening. However, using the invasive method there was a significant reduction in the risk of clinical worsening only. The Swedish/COMPERA method found a significant reduction in the risk of death and clinical worsening in the low-risk group compared with the intermediate-risk group at follow-up. Interestingly, the French non-invasive approach was recently found to be more accurate than the Swedish/COMPERA model in identifying patients with an excellent long-term survival [31]. In the present study, however, both tools seemed to have a similar predictive value. It is important to note that many patients remained in the intermediate-risk group at all time-points, and further research is necessary to provide additional discrimination and guidance on which patients in this category would benefit from intervention.

The results of the present analysis were consistent with previous studies of risk assessment in PAH. The RRS was developed as a simplified calculator for everyday clinical use to predict survival in patients with PAH, based on data from the REVEAL registry [32]. Studies have shown that the RRS has good discriminatory ability for predicting survival in patients with newly or previously diagnosed PAH and that changes in RRS over a 12-month period are also predictive of survival [32,33]. Assessment of the PATENT patient populations using the RRS showed results in line with the current post hoc analyses [34]. In patients with PAH, 


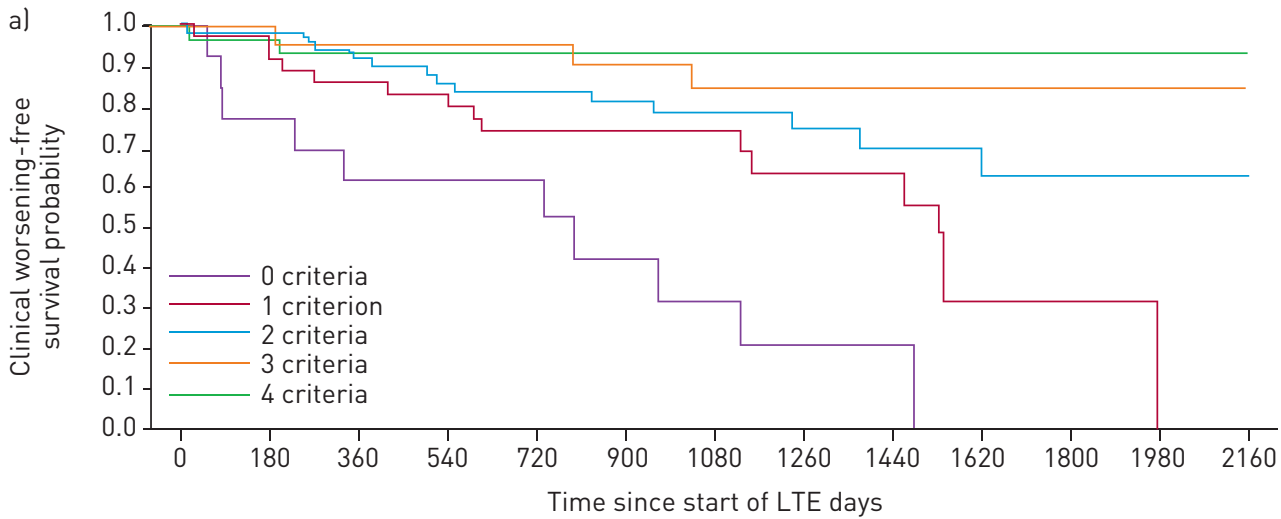

Patients at risk $\mathrm{n}$ :

$\begin{array}{ccccccccccccc}-14 & 10 & 8 & 7 & 7 & 4 & 3 & 2 & 2 & 0 & 0 & 0 & 0 \\ -37 & 34 & 29 & 28 & 23 & 17 & 14 & 10 & 8 & 4 & 3 & 0 & 0 \\ -50 & 48 & 45 & 41 & 38 & 30 & 25 & 19 & 12 & 10 & 8 & 4 & 2 \\ -31 & 30 & 27 & 25 & 24 & 22 & 18 & 16 & 14 & 11 & 7 & 2 & 1 \\ -23 & 22 & 21 & 21 & 21 & 19 & 15 & 13 & 10 & 9 & 6 & 4 & 3\end{array}$

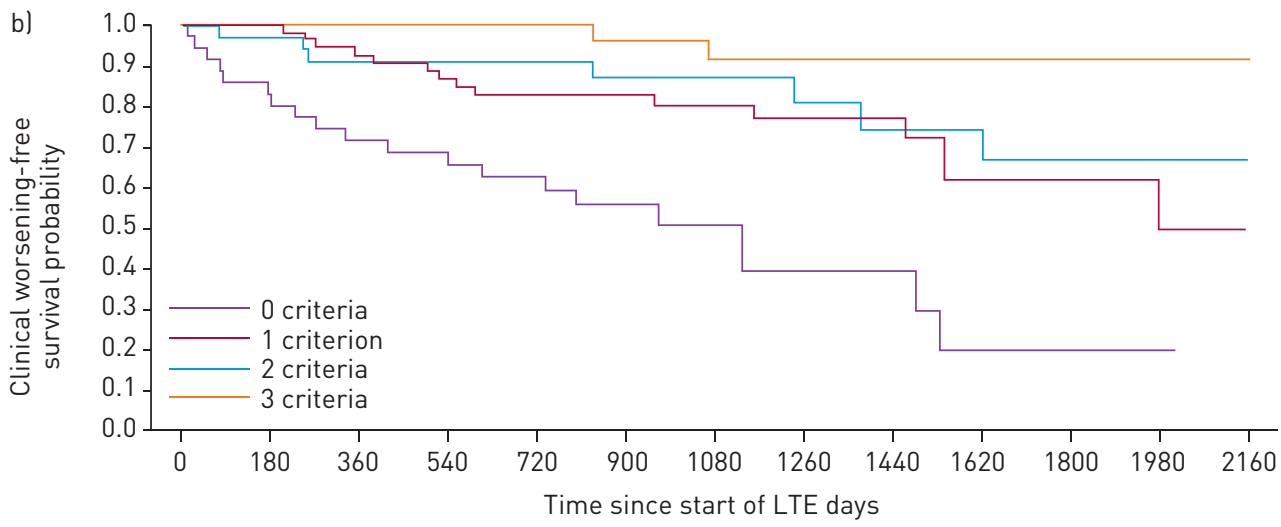

Patients at risk $\mathrm{n}$ :

$\begin{array}{lllllllllllll}-37 & 29 & 25 & 23 & 19 & 13 & 9 & 7 & 6 & 2 & 2 & 1 & 0 \\ -55 & 54 & 48 & 44 & 40 & 33 & 28 & 21 & 16 & 11 & 9 & 4 & 3 \\ -33 & 32 & 28 & 26 & 26 & 21 & 18 & 13 & 11 & 10 & 7 & 2 & 1 \\ -30 & 29 & 29 & 29 & 28 & 25 & 20 & 19 & 13 & 11 & 6 & 3 & 2\end{array}$

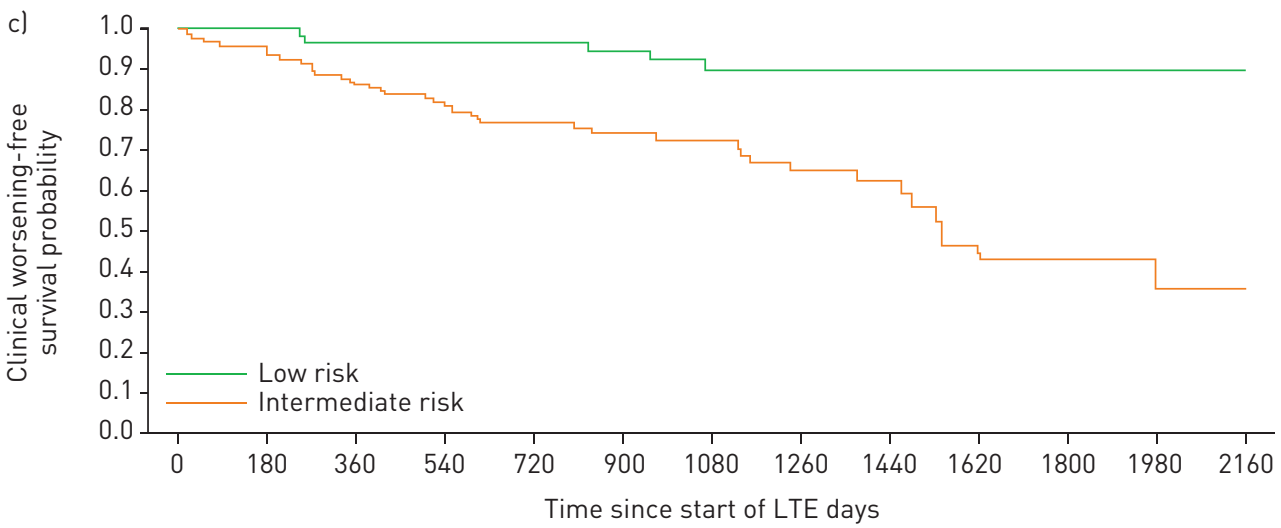

Patients at risk $\mathrm{n}$ :

$\begin{array}{lllllllllllll}-60 & 59 & 55 & 52 & 51 & 46 & 34 & 29 & 24 & 20 & 13 & 5 & 4 \\ -90 & 82 & 73 & 69 & 61 & 46 & 41 & 31 & 22 & 14 & 11 & 5 & 2\end{array}$


FIGURE 6 Kaplan-Meier curves for clinical worsening-free survival in patients based on risk stratification at CHEST-1 follow-up: a) French registry invasive method, b) French registry non-invasive method and c) Swedish/COMPERA method. Only patients receiving riociguat $2.5 \mathrm{mg}$ three times daily maximum in CHEST-1 who participated in CHEST-2 were considered in this analysis. Data were based on observed cases with no imputation. Day $0=$ start of extension study. Only five patients were in the Swedish/COMPERA high-risk group at CHEST-1 follow-up and were therefore not included in the analysis for this method. French registry invasive method: number of low-risk criteria fulfilled: 6-min walk distance (6MWD) $>440 \mathrm{~m}$, World Health Organization (WHO) functional class $(\mathrm{FC}) \mathrm{I} / \mathrm{II}$, right atrial pressure (RAP) $<8 \mathrm{mmHg}$ and cardiac index $\geqslant 2.5 \mathrm{~L} \cdot \mathrm{min}^{-1} \cdot \mathrm{m}^{-2}$. French registry non-invasive method: number of low-risk criteria fulfilled: $6 \mathrm{MWD}>440 \mathrm{~m}$, WHO FC I/II and N-terminal pro-brain natriuretic peptide (NT-proBNP) $<300 \mathrm{pg} \cdot \mathrm{mL}^{-1}$. Swedish/COMPERA method: mean of grades (1-3: low, intermediate, high) of six available criteria (6MWD, WHO FC, NT-proBNP, RAP, cardiac index and mixed venous oxygen saturation) as defined in the European Society of Cardiology/ European Respiratory Society 2015 treatment guidelines, rounded to the nearest integer. Log-rank test: invasive $p<0.0001$, non-invasive $p<0.0001$ and Swedish/COMPERA $p<0.0001$. LTE: long-term extension.

RRS and risk stratum were significantly improved by riociguat between baseline and follow-up. Moreover, RRS at baseline and at PATENT-1 follow-up, and change in RRS during PATENT-1, were all significantly associated with both survival and clinical worsening-free survival [34]. The RRS has been further refined recently to allow better risk delineation and include new clinically useful variables [35]. Another predictive equation that has been developed in PAH is that from the French Pulmonary Hypertension Network registry, which like the RRS and the risk assessment methods analysed in the present study has shown good discrimination and calibration for prediction of survival in patients with PAH [36, 37].

The results of the present analysis have a number of implications for clinical practice in the management of PAH. For example, registry findings with the French non-invasive method demonstrated that invasive measures may not always be essential for risk assessment. Our study also demonstrated discrimination between prognostic groups for both survival and clinical worsening-free survival using the French noninvasive method at both baseline and PATENT-1 follow-up, while the discrimination ability of the French invasive method was limited to just clinical worsening-free survival. This is also true of the RRS, which does not require haemodynamic parameters for calculation [33]. In clinical practice, it is important that individual patient risk should be assessed by experienced treating physicians, as patients may achieve a low-risk category for some parameters and not others. However, while the ESC/ERS risk stratification model allows for comprehensive assessment of patients with PAH $[1,12]$, the current post hoc analysis suggests that as few as three non-invasive parameters may be of use for initial patient risk assessment, potentially before a more comprehensive assessment takes place. Importantly, the use of abbreviated tools in clinical practice is still under discussion and until further studies are undertaken, clinicians should use currently available risk assessment tools, finding the best balance of discriminatory power and ease of use for making treatment decisions for the individual patient. Another way in which these tools could be used, as demonstrated by the association of patient risk group or stratum with long-term outcomes shown in our analyses and the registry analyses, is in the development of new, goal-oriented clinical trial end-points. However, this needs to be validated in the context of a prospective clinical study [29].

Risk assessment is more challenging in patients with CTEPH than in those with PAH due to the multiple treatment options available for CTEPH, including PEA, balloon pulmonary angioplasty and medical therapy. Furthermore, there may be prognostic factors specific to CTEPH that are not included in the existing risk models. However, each of the three abbreviated tools in the current post hoc analysis had the ability to discriminate prognosis for survival and clinical worsening-free survival among patients with inoperable or persistent/recurrent CTEPH following 16 weeks of treatment with riociguat, although further analyses would need to be performed to statistically compare the discriminatory ability of the tools. These observations are in line with similar results found when the RRS was applied to the CHEST study database, in which riociguat significantly improved RRS and risk stratum versus placebo from baseline to week 16 [16]. In addition, RRS at baseline and week 16, and change in RRS during CHEST-1, were significantly associated with survival and clinical worsening-free survival over 2 years in CHEST-2. Further study of the challenges and tools for assessing risk in CTEPH is warranted with multiple therapeutic options used alone, sequentially or in combination [1,38]. A recent study has shown that the Swedish/ COMPERA model can be used to predict mortality in patients with non-operated CTEPH [17]; however, it may be more appropriate to develop a risk assessment tool specific to CTEPH and test it in contemporary cohorts of patients recruited in the modern management era.

Limitations of the ESC/ERS risk assessment model, also common to the abbreviated models presented here, include the lack of weighting of the various risk criteria and the reliance on expert consensus regarding parameter inclusion, rather than on results of statistical modelling [39]. Although the thresholds used for the abbreviated risk models could statistically discriminate patient cohorts for outcome, 
Hazard ratio p-value b]

(95\% CI)

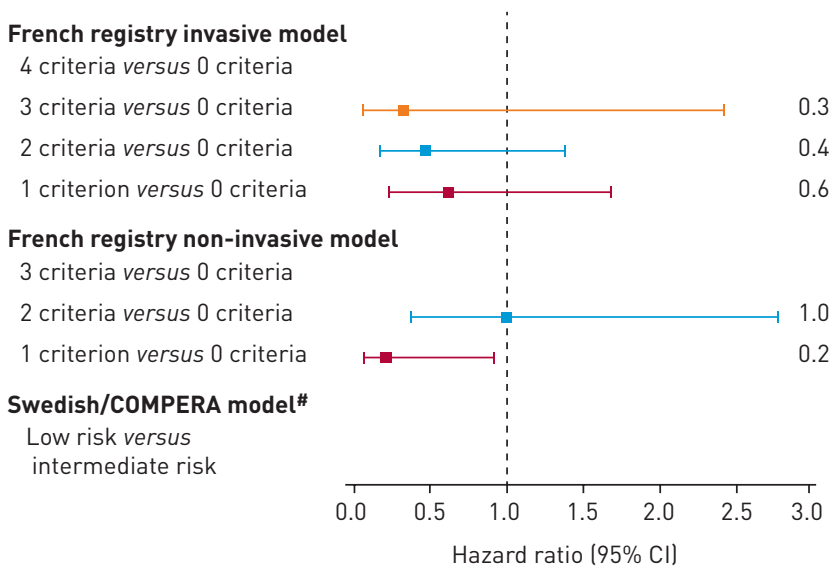

c)

French registry invasive mode

4 criteria versus 0 criteria

3 criteria versus 0 criteria

2 criteria versus 0 criteria

1 criterion versus 0 criteria

\section{French registry non-invasive model}

3 criteria versus 0 criteria

2 criteria versus 0 criteria

1 criterion versus 0 criteria

\section{Swedish/COMPERA model ${ }^{\#}$} Low risk versus intermediate risk

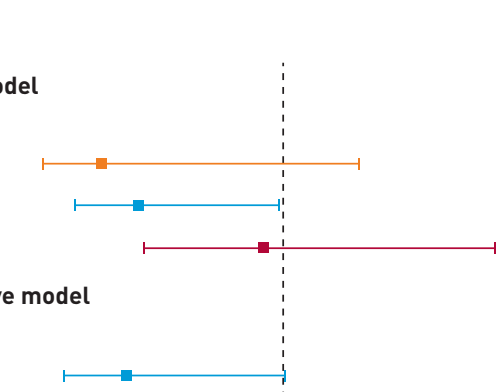

\begin{tabular}{|c|c|c|}
\hline \multirow{2}{*}{$\begin{array}{l}\text { Hazard ratio } \\
\qquad(95 \% \mathrm{Cl})\end{array}$} & \multirow[t]{2}{*}{$\mathrm{p}$-value } & \multirow{2}{*}{$\begin{array}{l}\text { d) } \\
\text { French registry invasive mo } \\
4 \text { criteria versus } 0 \text { criteria }\end{array}$} \\
\hline & & \\
\hline 00 (0.069-1.299) & 0.107 & 3 criteria versus 0 criteria \\
\hline $38(0.194-0.988)$ & 0.047 & 2 criteria versus 0 criteria \\
\hline $19(0.463-1.824)$ & 0.809 & 1 criterion versus 0 criteria \\
\hline & & $\begin{array}{l}\text { French registry non-invasive } \\
3 \text { criteria versus } 0 \text { criteria }\end{array}$ \\
\hline $91(0.152-1.007)$ & 0.052 & 2 criteria versus 0 criteria \\
\hline $72(0.170-0.811)$ & 0.013 & 1 criterion versus 0 criteria \\
\hline & & Swedish/COMPERA mo \\
\hline ar & 0.035 & $\begin{array}{l}\text { Low risk versus } \\
\text { intermediate risk }\end{array}$ \\
\hline
\end{tabular}

$\begin{array}{lllllllllll}0.0 & 0.2 & 0.4 & 0.6 & 0.8 & 1.0 & 1.2 & 1.4 & 1.6 & 1.8 & 20\end{array}$ intermediate risk 3 criteria versus 0 criteria $0.459(0.153-1.376)-0.165-2$ criteria versus 0 criteria $0.601(0.214-1.693) \quad 0.336 \quad 1$ criterion versus 0 criteria

French registry non-invasive mode

3 criteria versus 0 criteria

2 criteria versus 0 criteria

$0.205(0.047-0.906) \quad 0.037 \quad 1$ criterion versus 0 criteria

Swedish/COMPERA model ${ }^{\#}$

Low risk versus

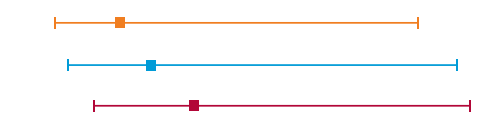

$\longmapsto$

$\begin{array}{rrrrrrrrrrr}0.0 & 0.1 & 0.2 & 0.3 & 0.4 & 0.5 & 0.6 & 0.7 & 0.8 & 0.9 & 1.0\end{array}$

Hazard ratio $(95 \% \mathrm{Cl})$

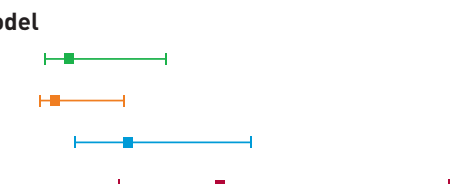

p-value $(95 \% \mathrm{Cl})$

$0.070(0.019-0.260) \quad<0.001$

$0.038(0.008-0.178)<0.001$

$0.185(0.080-0.430) \quad<0.001$

$0.371 \quad(0.166-0.826) \quad 0.015$

$0.061 \quad(0.014-0.262) \quad<0.001$

$0.233(0.098-0.557) \quad 0.001$

$0.313(0.159-0.617)<0.001$

$0.164(0.064-0.420)<0.001$

$\begin{array}{lllllllllll}0.0 & 0.1 & 0.2 & 0.3 & 0.4 & 0.5 & 0.6 & 0.7 & 0.8 & 0.9 & 1.0\end{array}$ Hazard ratio $(95 \% \mathrm{CI})$

FIGURE 7 Forest plots comparing survival in CHEST-2 expressed as hazard ratios (95\% CI) for mortality comparing patients who achieved one or more low-risk criteria compared with those who achieved no low-risk criteria at a) baseline and b) follow-up in CHEST-1, and clinical worsening-free survival in CHEST-2 expressed as hazard ratios (95\% CI) for clinical worsening comparing patients who achieved one or more low-risk criteria compared with those who achieved no low-risk criteria at c) baseline and d) follow-up in CHEST-1. Only patients receiving riociguat $2.5 \mathrm{mg}$ three times daily maximum in CHEST-1 who participated in CHEST-2 were considered in this analysis. French registry invasive method: number of low-risk criteria fulfilled: 6-min walk distance (6MWD) $>440 \mathrm{~m}$. World Health Organization (WHO) functional class (FC) I/II, right atrial pressure (RAP) $<8 \mathrm{mmHg}^{2}$ and cardiac index $\geqslant 2.5 \mathrm{~L} \cdot \mathrm{min}^{-1}$. $\mathrm{m}^{-2}$. French registry non-invasive method: number of low-risk criteria fulfilled: 6MWD $>440 \mathrm{~m}$, WHO FC I/II and N-terminal pro-brain natriuretic peptide (NT-proBNP) <300 pg-mL ${ }^{-1}$. Swedish/COMPERA method: mean of grades (1-3: low, intermediate, high) of six available criteria (6MWD, WHO FC, NT-proBNP, RAP, cardiac index and mixed venous oxygen saturation) as defined in the European Society of Cardiology/European Respiratory Society 2015 treatment guidelines, rounded to the nearest integer. ${ }^{\#}$ : due to the small number of patients in the high-risk category at baseline Society of Cardiology/European Respiratory Society 2015 treatment guidelines, rounded to the nearest integer. \#: due to the small number of patients in the high-risk category at baseline
and follow-up according to the Swedish/COMPERA method, these patients were not included in this analysis. Due to no or few events in some risk groups/strata, hazards ratios could not be and follow-up according to 
the statistical assessment of patient groups may be of limited value for the individual patient. Furthermore, the ESC/ERS low-risk thresholds used here have been identified using regression analyses in specific study populations and therefore may not be as useful in other patient populations. A further limitation of the current post hoc analysis is the lack of C-indices, which were not calculated, meaning that the methods cannot be compared statistically. It should also be noted that there were differences between the patient populations in the different analyses used in the present study. For example, a far higher percentage of patients in the French non-invasive assessment had one or more low-risk criteria than were identified as "low risk" in the Swedish/COMPERA method. While it may not therefore be possible to compare specific data directly between the different methods, the overall results for each method were broadly similar. In addition, the patient numbers in some subgroups were very small, including the cohorts with zero low-risk criteria. This, combined with the low numbers of events (deaths or clinical worsening events), resulted in large confidence intervals, increasing the uncertainty of the results. Finally, the analysis excluded patients who did not complete the original PATENT-1/CHEST-1 studies. As long-term outcomes in these patients may have been worse than those who completed the original studies and were included in the analysis, this may have affected our findings.

In conclusion, this post hoc analysis confirms the results of previous registry evaluations when each abbreviated model was applied to a prospective pivotal clinical study database of mostly prevalent PAH patients. Achieving one or more low-risk criteria using the French non-invasive method or a low-risk stratum according to the Swedish/COMPERA method at follow-up conferred a significantly reduced risk of death or experiencing a clinical worsening event. Observations in patients with inoperable and persistent/ recurrent CTEPH demonstrated the potential utility of PAH risk prediction tools in this population.

Acknowledgements: Editorial support was provided by Adelphi Communications Ltd (Bollington, UK), supported by Bayer AG.

Author contributions: M. Humbert conceived the idea for the analyses detailed in this manuscript. R.L. Benza and H.W. Farber were also involved in the conceptualisation of these analyses in the PATENT and CHEST study databases. M. Humbert, H.W. Farber, H-A. Ghofrani, M.M. Hoeper and C. Meier contributed to the design and data collection in the PATENT and CHEST studies. D. Busse undertook statistical analyses of the data in the manuscript. All authors contributed to drafting and critical review of the manuscript. All authors approved the manuscript for submission.

Conflict of interest: H.W. Farber reports grants from Actelion, Gilead and United Therapeutics, personal fees from Actelion, Bayer AG, Bellerophon, Gilead and United Therapeutics, during the conduct of the study. H-A. Ghofrani reports grants and personal fees from Actelion, Bayer AG, Ergonex and Pfizer, personal fees from Gilead, GSK, Merck and Novartis, outside the submitted work. R.L. Benza reports grants from Bellerophon, Bayer AG, Actelion and EIGER, during the conduct of the study. D. Busse was an external employee of Bayer AG, during the conduct of the study. C. Meier was an employee of Bayer AG, during the conduct of the study. M.M. Hoeper reports consultancy fees from Actelion, Bayer AG, GSK and Pfizer, during the conduct of the study. M. Humbert reports personal fees from Bayer and Merck, during the conduct of the study; personal fees from Actelion, and support from GSK, Johnson and Johnson and United Therapeutics, outside the submitted work.

Support statement: The PATENT and CHEST studies were funded by Bayer AG. Funding information for this article has been deposited with the Crossref Funder Registry.

\section{References}

1 Galiè N, Humbert M, Vachiery JL, et al. 2015 ESC/ERS Guidelines for the diagnosis and treatment of pulmonary hypertension: The joint task force for the diagnosis and treatment of pulmonary hypertension of the European Society of Cardiology (ESC) and the European Respiratory Society (ERS): Endorsed by: Association for European Paediatric and Congenital Cardiology (AEPC), International Society for Heart and Lung Transplantation (ISHLT). Eur Respir J 2015; 46: 903-975.

2 McLaughlin VV, Archer SL, Badesch DB, et al. ACCF/AHA 2009 expert consensus document on pulmonary hypertension: a report of the American College of Cardiology Foundation Task Force on Expert Consensus Documents and the American Heart Association: developed in collaboration with the American College of Chest Physicians; American Thoracic Society, Inc.; and the Pulmonary Hypertension Association. J Am Coll Cardiol 2009; 53: 1573-1619.

3 Ghofrani HA, Humbert M, Langleben D, et al. Riociguat: mode of action and clinical development in pulmonary hypertension. Chest 2017; 151: 468-480.

4 Jenkins D. PEA: a potentially curative treatment option for patients with CTEPH. Eur Respir Rev 2015; 24: 263-271.

5 Kim NH, Delcroix M, Jenkins DP, et al. Chronic thromboembolic pulmonary hypertension. J Am Coll Cardiol 2013; 62: 25 Suppl., D92-D99.

6 Freed DH, Thomson BM, Berman M, et al. Survival after pulmonary thromboendarterectomy: effect of residual pulmonary hypertension. J Thorac Cardiovasc Surg 2011; 141: 383-387.

7 Lang IM. Managing chronic thromboembolic pulmonary hypertension: pharmacological treatment options. Eur Respir Rev 2009; 111: 24-28. 
8 Mayer E, Jenkins D, Lindner J, et al. Surgical management and outcome of patients with chronic thromboembolic pulmonary hypertension: results from an international prospective registry. J Thorac Cardiovasc Surg 2011; 141: 702-710.

9 Wilkens H, Lang I, Behr J, et al. Chronic thromboembolic pulmonary hypertension (CTEPH): updated recommendations of the Cologne Consensus Conference 2011. Int J Cardiol 2011; 154: Suppl. 1, S54-S60.

10 Cannon JE, Su L, Kiely DG, et al. Dynamic risk stratification of patient long-term outcome after pulmonary endarterectomy: results from the UK national cohort. Circulation 2016; 133: 1761-1771.

11 Kramm T, Wilkens H, Fuge J, et al. Incidence and characteristics of chronic thromboembolic pulmonary hypertension in Germany. Clin Res Cardiol 2018; 107: 548-553.

12 Galiè N, Channick RN, Frantz RP, et al. Risk stratification and medical therapy of pulmonary arterial hypertension. Eur Respir J 2019; 53: 1801889.

13 Boucly A, Weatherald J, Savale L, et al. Risk assessment, prognosis and guideline implementation in pulmonary arterial hypertension. Eur Respir J 2017; 50: 1700889.

14 Hoeper MM, Kramer T, Pan Z, et al. Mortality in pulmonary arterial hypertension: prediction by the 2015 European pulmonary hypertension guidelines risk stratification model. Eur Respir J 2017; 50: 1700740.

15 Kylhammar D, Kjellstrom B, Hjalmarsson C, et al. A comprehensive risk stratification at early follow-up determines prognosis in pulmonary arterial hypertension. Eur Heart J 2018; 39: 4175-4181.

16 Benza RL, Farber HW, Frost A, et al. REVEAL risk score in patients with chronic thromboembolic pulmonary hypertension receiving riociguat. J Heart Lung Transplant 2018; 37: 836-843.

17 Delcroix M, Staehler G, Gall H, et al. Risk assessment in medically treated chronic thromboembolic pulmonary hypertension patients. Eur Respir J 2018; 52: 1800248.

18 Stasch JP, Pacher P, Evgenov OV. Soluble guanylate cyclase as an emerging therapeutic target in cardiopulmonary disease. Circulation 2011; 123: 2263-2273.

19 Stasch JP, Evgenov OV. Soluble guanylate cyclase stimulators in pulmonary hypertension. Handb Exp Pharmacol 2013; 218: 279-313.

20 Humbert M, Ghofrani HA. The molecular targets of approved treatments for pulmonary arterial hypertension. Thorax 2016; 71: 73-83.

21 Follmann M, Griebenow N, Hahn MG, et al. The chemistry and biology of soluble guanylate cyclase stimulators and activators. Angew Chem Int Ed Engl 2013; 52: 9442-9462.

22 Schermuly RT, Stasch JP, Pullamsetti SS, et al. Expression and function of soluble guanylate cyclase in pulmonary arterial hypertension. Eur Respir J 2008; 32: 881-891.

23 Ghofrani HA, D'Armini AM, Grimminger F, et al. Riociguat for the treatment of chronic thromboembolic pulmonary hypertension. N Engl J Med 2013; 369: 319-329.

24 Ghofrani HA, Galiè N, Grimminger F, et al. Riociguat for the treatment of pulmonary arterial hypertension. N Engl J Med 2013; 369: 330-340.

25 Simonneau G, D'Armini AM, Ghofrani HA, et al. Riociguat for the treatment of chronic thromboembolic pulmonary hypertension: a long-term extension study (CHEST-2). Eur Respir J 2015; 45: 1293-1302.

26 Ghofrani HA, Grimminger F, Grunig E, et al. Predictors of long-term outcomes in patients treated with riociguat for pulmonary arterial hypertension: data from the PATENT-2 open-label, randomised, long-term extension trial. Lancet Respir Med 2016; 4: 361-371.

27 Simonneau G, D'Armini AM, Ghofrani HA, et al. Predictors of long-term outcomes in patients treated with riociguat for chronic thromboembolic pulmonary hypertension: data from the CHEST-2 open-label, randomised, long-term extension trial. Lancet Respir Med 2016; 4: 372-380.

28 Rubin LJ, Galiè N, Grimminger F, et al. Riociguat for the treatment of pulmonary arterial hypertension: a long-term extension study (PATENT-2). Eur Respir J 2015; 45: 1303-1313.

29 Weatherald J, Boucly A, Sahay S, et al. The low-risk profile in pulmonary arterial hypertension: time for a paradigm shift to goal-oriented clinical trial endpoints? Am J Respir Crit Care Med 2017; 197: 860-868.

30 Humbert $\mathrm{M}$, Ghofrani H-A, Busse D, et al. Riociguat in pulmonary arterial hypertension: ERS/ESC risk assessment in PATENT. Eur Respir J 2016; 48: Suppl. 60, PA2401.

31 Hoeper MM, Pittrow D, Opitz C, et al. Risk assessment in pulmonary arterial hypertension. Eur Respir J 2018; 51: 1702606.

32 Benza R, Gomberg-Maitland M, Elliott CG, et al. Predicting survival in patients with pulmonary arterial hypertension: the REVEAL 2.0 risk score calculator in pulmonary arterial hypertension. Chest 2019; in press [https://doi.org/10.1016/j.chest.2019.02.004].

33 Benza RL, Miller DP, Foreman AJ, et al. Prognostic implications of serial risk score assessments in patients with pulmonary arterial hypertension: a Registry to Evaluate Early and Long-Term Pulmonary Arterial Hypertension Disease Management (REVEAL) analysis. J Heart Lung Transplant 2015; 34: 356-361.

34 Benza RL, Farber HW, Frost A, et al. REVEAL risk scores applied to riociguat-treated patients in PATENT-2: impact of changes in risk score on survival. J Heart Lung Transplant 2017; 37: 513-519.

35 Benza RL, Elliot CG, Farber HW, et al. Updated risk score calculator for pulmonary arterial hypertension patients. J Heart Lung Transplant 2017; 36: S19.

36 Humbert M, Sitbon O, Chaouat A, et al. Survival in patients with idiopathic, familial, and anorexigen-associated pulmonary arterial hypertension in the modern management era. Circulation 2010; 122: 156-163.

37 Sitbon O, Benza RL, Badesch DB, et al. Validation of two predictive models for survival in pulmonary arterial hypertension. Eur Respir J 2015; 46: 152-164.

38 Madani M, Ogo T, Simonneau G. The changing landscape of chronic thromboembolic pulmonary hypertension management. Eur Respir Rev 2017; 26: 170105.

39 Benza RL, Farber HW, Selej M, et al. Assessing risk in pulmonary arterial hypertension: what we know, what we don't. Eur Respir J 2017; 50: 1701353. 